\title{
Zu diesem Heft
}

Angesichts der immer weiter voranschreitenden Ökonomisierung und Globalisierung der Medien plädiert Matthias Karmasin in seinem Artikel „Medienethik als Wirtschaftsethik medialer Kommunikation?" für eine Medienethik, in die auch die Ergebnissse der aktuellen wirtschaftsethischen Diskussionen integriert werden. Karmasin skizziert ein Konzept der Medienethik als Wirtschaftsethik bzw. als Ethik der Medienunternehmung vom Ansatz des sog. Stakeholder Managements her und formuliert vor diesem Hintergrund Rahmenbedingungen und Möglichkeiten der Institutionalisierung einer Ethik der Medienunternehmung.

Communicatio Socialis wird auch in den folgenden Heften schwerpunktmäßig immer wieder medienethische Fragestellungen aufgreifen. Der Aufsatz von Matthias Karmasin eröffnet diese Artikelreihe zur Medienethik.

Auch wenn der Beitrag von Michael Krzeminski „Medien in der Entwicklungspolitik. Alte Paradigmen und neue Perspektiven" sich nicht direkt mit medienethischen Problemen beschäftigt, so wären doch die ethischen und auch medienethischen Implikationen der Antwort auf die Frage, welche Rolle die Medien im Entwicklungsprozeß spielen, leicht aufzuzeigen. Mit dieser Thematik befaßt sich nämlich der Artikel von Michael Krzeminski. Er stellt dar, wie die letzten vierzig Jahre Forschungstätigkeit von einigen wenigen Theorieansätzen dominiert wurden. Sie beruhen auf Denkmodellen, die in starker Abhängigkeit von der jeweiligen entwicklungspolitischen Diskussion und von der Theorieentwicklung in den Kommunikationswissenschaften entstanden sind. Zu einer eigenständigen Theorie der Entwicklungskommunikation ist es nach Krzeminski bisher nicht gekommen. Vor diesem Hintergrund plädiert Krzeminski für den Abschied von der Vorstellung einer medialen Gesamtversorgung von Kommunikationsräumen und für die Konzentration auf die speziellen Kommunikationsbedürfnisse einheimischer Akteure, die empirisch jeweils zu ermitteln wären.

Über Erfahrungen mit der Einrichtung eines „Presse-Ombudsmanns“ in den Niederlanden sowie in Schweden, in den USA und in der Schweiz informiert Huub Evers. Jean Marie Brunot berichtet über die Entwicklung der von den Assumptionisten vor über 100 Jahren in Frankreich gegründeten „Bayard Press“ hin zu einem modernen katholischen Medienkonzern und Ferdinand Oertel stellt den größten Dienstleister für die katholische Presse in den USA vor, die "Catholic Press Association (CPA)".

In zwei knappen Berichten schildern Verena Hanf und Alexandru Mi- 
hailescu die Entwicklung der rumänischen Medien nach 1989 und bilanzieren unter dem Stichwort "Papamania" das Medienecho in Rumänien auf den Besuch Papst Johannes Paul II. vom 7.-9. Mai 1999 in Bukarest.

Vom 20.-23. Juli 1999 fand an der Universität Edinburgh eine internationale Konferenz über Medien, Religion und Kultur statt, über deren Verlauf und Ergebnisse Franz-Josef Eilers informiert.

Der Dokumentationsteil enthält die Erklärung des Vorsitzenden der Publizistischen Kommission der Deutschen Bischofskonferenz, Bischof Dr. Hermann Josef Spital, "Leselust und Lesenutzen" und das Orientierungspapier "Consultation on Evangelization and Communication. Orientation and Recommendations for the Church of Asia", das auf einem vom Sekretariat für Evangelisation und Soziale Kommunikation der Asiatischen Bischofskonferenz organisierten Treffen in Chennai (Indien) verabschiedet wurde. 